Supporting Information

\title{
Lipopolysaccharide Neutralization by Cationic-amphiphilic Polymers through Pseudo-aggregate Formation
}

Divakara SSM Uppu and Jayanta Haldar*

Chemical Biology \& Medicinal Chemistry Laboratory, New Chemistry Unit (NCU), Jawaharlal Nehru Centre for Advanced Scientific Research (JNCASR), Jakkur, Bengaluru, Karnataka, India-560064.

*Corresponding author. E-mail: jayanta@jncasr.ac.in 


\section{Materials and Methods}

All the solvents were of reagent grade and dried prior to use wherever required. Poly(isobutylene-alt-maleic anhydride) $\left(\mathrm{M}_{\mathrm{w}} \sim 6000\right.$ Da, Catalog no. 531278) purchased from Sigma-Aldrich and used as received. All other reagents were procured from the commercial suppliers and used as received. Culture media and the antibiotics were from HIMEDIA (India) and Sigma-Aldrich respectively. NMR spectra were recorded using Bruker AMX-400 (400 MHz

for ${ }^{1} \mathrm{H}$ and $100 \mathrm{MHz}$ for ${ }^{13} \mathrm{C}$ ) spectrometer. The chemical shifts $(\delta)$ are reported in parts per million downfield from the peak for the internal standard TMS for ${ }^{1} \mathrm{H}-\mathrm{NMR}$. Infrared (IR) spectra of the solid compounds were recorded on Bruker IFS66 V/s spectrometer using $\mathrm{KBr}$ pellets. IR spectra of the compounds soluble in low-boiling solvents were recorded with the same instrument using $\mathrm{NaCl}$ crystal. Mass spectra were recorded on a Micromass Q-ToF micromass spectrometer. Optical density and absorbance were measured by Tecan InfinitePro series M200 Microplate Reader.

\section{Synthesis of side chain alkylating agents}

N-alkyl-1-bromoethanamide: Alkyl amine $(118 \mathrm{mmol})$ was dissolved in dichloromethane (55 $\mathrm{mL})$. Potassium carbonate, $\mathrm{K}_{2} \mathrm{CO}_{3}(24.55 \mathrm{~g}, 178 \mathrm{mmol})$ was dissolved in $60 \mathrm{~mL}$ of distilled water and the solution was added to the organic solution. The resulting two phase solution was cooled to $4{ }^{\circ} \mathrm{C}$. A solution of bromoacetyl bromide $(35.85 \mathrm{~g}, 178 \mathrm{mmol})$ in dichloromethane $(55$ $\mathrm{mL}$ ) was carefully added drop wise to the cooled solution while maintaining the temperature at 4 ${ }^{\circ} \mathrm{C}$ for about $30 \mathrm{~min}$. Then the reaction mixture was stirred at room temperature for $12 \mathrm{~h}$. The aqueous solution was separated and washed with dichloromethane $(2 \times 25 \mathrm{~mL})$. The organic 
solution was washed with water $(2 \times 50 \mathrm{~mL})$ and passed over the anhydrous $\mathrm{Na}_{2} \mathrm{SO}_{4}$ and concentrated to yield a white solid quantitatively (100\% yield).

N-alkyl-1-bromoethanoate: 1-propanol (116.5 mmol) was dissolved in dichloromethane (55 $\mathrm{mL})$. Potassium carbonate, $\mathrm{K}_{2} \mathrm{CO}_{3}(19.32 \mathrm{~g}, 140 \mathrm{mmol})$ was dissolved in $60 \mathrm{~mL}$ of distilled water and the solution was added to the organic solution. The resulting two phase solution was cooled to $4{ }^{\circ} \mathrm{C}$. A solution of bromoacetyl bromide $(28.21 \mathrm{~g}, 140 \mathrm{mmol})$ in dichloromethane (55 $\mathrm{mL}$ ) was carefully added drop wise to the cooled solution while maintaining the temperature at 4 ${ }^{\circ} \mathrm{C}$ for about $30 \mathrm{~min}$. Then the reaction mixture was stirred at room temperature for $12 \mathrm{~h}$. The aqueous solution was separated and washed with dichloromethane $(2 \times 25 \mathrm{~mL})$. The organic solution was washed with water $(2 \times 50 \mathrm{~mL})$ and passed over the anhydrous $\mathrm{Na}_{2} \mathrm{SO}_{4}$ and concentrated to yield an oily liquid quantitatively.

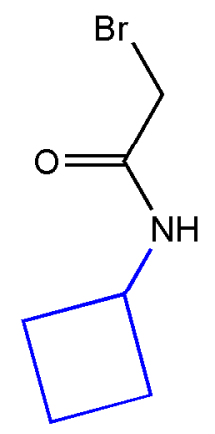

N-(cyclobutyl)-1-bromoethanamide: FT-IR: $3250 \mathrm{~cm}^{-1}$ (amide N-H str.), 2950-2850 cm-1 (C-H str.), $1680 \mathrm{~cm}^{-1}$ (Amide I, C=O str.), $1560 \mathrm{~cm}^{-1}$ (Amide II, N-H ben.), 1470-1410 $\mathrm{cm}^{-1}$ (C-C str.), 1290-1110 cm ${ }^{-1}$ (C-O str.); ${ }^{1} \mathrm{H}-\mathrm{NMR}\left(400 \mathrm{MHz}, \mathrm{CDCl}_{3}\right.$ ): $\delta / \mathrm{ppm} 1.747$ (m, cyCH $\left.2,2 \mathrm{H}\right), 1.924$ (m, $\left.\mathrm{cyCH}_{2}, 2 \mathrm{H}\right), 2.357$ (m, $\left.\mathrm{cyCH}_{2}, 2 \mathrm{H}\right), 3.843$ (s, - $\left.\mathrm{COCH}_{2} \mathrm{Br}, 2 \mathrm{H}\right), 4.373$ (m, суCH, 1H), 6.576 (br s, amide-NHCO, 1H) ); HR-MS: m/z 192.00 (observed): 191.99 (calculated for $[\mathrm{M}+\mathrm{H}]^{+}$). 


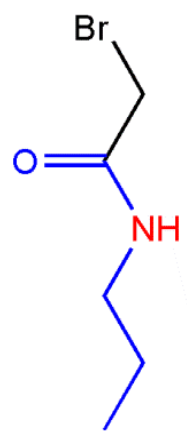

N-(n-propyl)-1-bromoethanamide: FT-IR: $3250 \mathrm{~cm}^{-1}$ (amide N-H str.), 2950-2850 (C-H str.), $1680 \mathrm{~cm}^{-1}$ (Amide I, $\mathrm{C}=\mathrm{O}$ str.), $1560 \mathrm{~cm}^{-1}$ (Amide II, N-H ben.), 1470-1410 $\mathrm{cm}^{-1}$ (C-C str.), 1290-1110 (C-O str.); ${ }^{1} \mathrm{HNMR}\left(400 \mathrm{MHz}, \mathrm{CDCl}_{3}\right.$ ): $\delta / \mathrm{ppm} 0.878$ (t, terminal $-\mathrm{CH}_{3}, 3 \mathrm{H}$ ), 1.543 (m, - $\left.\mathrm{CONHCH}_{2} \mathrm{CH}_{2} \mathrm{CH}_{3}-, 2 \mathrm{H}\right), 3.278\left(\mathrm{t},-\mathrm{CONHCH}_{2}-, 2 \mathrm{H}\right), 3.881$ (s, - $\left.\mathrm{COCH}_{2} \mathrm{Br}, 2 \mathrm{H}\right), 6.475$ (br s, amide $-N H C O, 1 \mathrm{H})) ;{ }^{13} \mathrm{C}$ NMR $\left(100 \mathrm{MHz}, \mathrm{CDCl}_{3}\right): \delta 14.195,22.768,26.904,29.324$, 29.423, 29.588, 29.646, 29.708, 31.995, 40.403, 165.589; HR-MS: $m / z 180.00$ (observed): 179.99 (calculated for $[\mathrm{M}+\mathrm{H}]^{+}$).

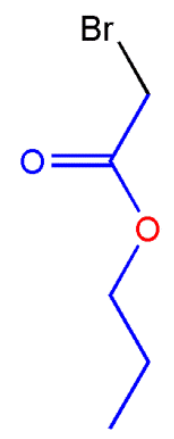

Propyl-1-bromoethanoate: FT-IR: 2950-2850 (C-H str.), $1735 \mathrm{~cm}^{-1}$ (C=O str.), 1470-1410 $\mathrm{cm}^{-1}$ (C-C str.), 1290-1110 (C-O str.); ${ }^{1} \mathrm{HNMR}$ (400 MHz, $\mathrm{CDCl}_{3}$ ): $\delta / \mathrm{ppm} 0.85$ (t, terminal $-\mathrm{CH}_{3}$, $3 \mathrm{H}), 1.57\left(\mathrm{~m},-\mathrm{COOCH}_{2} \mathrm{CH}_{2} \mathrm{CH}_{3}, 2 \mathrm{H}\right), 4.0\left(\mathrm{t},-\mathrm{COOCH}_{2}, 2 \mathrm{H}\right), 3.7\left(\mathrm{~s},-\mathrm{COCH}_{2} \mathrm{Br}, 2 \mathrm{H}\right) ;{ }^{13} \mathrm{C}$ NMR (100 MHz, $\left.\mathrm{CDCl}_{3}\right): \delta 14.195,22.768,26.904,29.324,29.423,29.588,29.646,29.708$, 31.995, 40.403, 171.19; HR-MS: $m / z 180.10$ (observed): 179.98 (calculated for $\mathrm{M}^{+}$). 


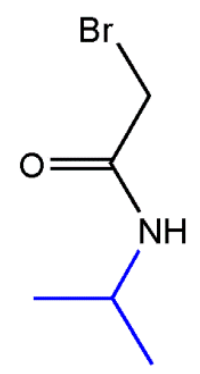

N-(isopropyl)-1-bromoethanamide: FT-IR: $3250 \mathrm{~cm}^{-1}$ (amide N-H str.), 2950-2850 (C-H str.), $1680 \mathrm{~cm}^{-1}$ (Amide I, $\mathrm{C}=\mathrm{O}$ str.), $1560 \mathrm{~cm}^{-1}$ (Amide II, N-H ben.), $1470-1410 \mathrm{~cm}^{-1}$ (C-C str.), 1290-1110 (C-O str.); ${ }^{1} \mathrm{HNMR}\left(400 \mathrm{MHz}, \mathrm{CDCl}_{3}\right): \delta / \mathrm{ppm} 1.19$ (d, $\left.-\mathrm{CH}\left(\mathrm{CH}_{3}\right)_{2}, 6 \mathrm{H}\right), 3.84$ (s, $\left.\mathrm{COCH}_{2} \mathrm{Br}, 2 \mathrm{H}\right), 4.06\left(\mathrm{~m},-\mathrm{CH}\left(\mathrm{CH}_{3}\right)_{2}, 1 \mathrm{H}\right), 6.286(\mathrm{br} \mathrm{s}$, amide $\left.-\mathrm{NHCO}, 1 \mathrm{H})\right)$; HR-MS: $m / z$ 180.00 (observed): 179.99 (calculated for $[\mathrm{M}+\mathrm{H}]^{+}$).

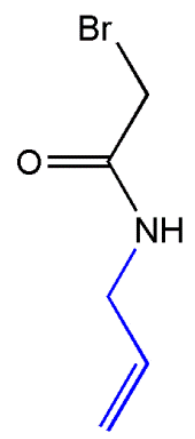

N-(prop-2-enyl)-1-bromoethanamide: FT-IR: $3250 \mathrm{~cm}^{-1}$ (amide N-H str.), $3049 \mathrm{~cm}^{-1}$ (C-H str.), $1680 \mathrm{~cm}^{-1}$ (Amide I, $\mathrm{C}=\mathrm{O}$ str.), $1642 \mathrm{~cm}^{-1}$ ( $\mathrm{C}=\mathrm{C}$ str.), $1560 \mathrm{~cm}^{-1}$ (Amide II, N-H ben.), 1470$1410 \mathrm{~cm}^{-1}$ (C-C str.), $1290-1110 \mathrm{~cm}^{-1}$ (C-O str.); ${ }^{1} \mathrm{H}-\mathrm{NMR}\left(400 \mathrm{MHz}, \mathrm{CDCl}_{3}\right.$ ): $\delta / \mathrm{ppm} 3.90$ (s, $\left.\mathrm{COCH}_{2} \mathrm{Br}, 2 \mathrm{H}\right), 3.93\left(\mathrm{dt},-\mathrm{CONHCH}_{2}-, 2 \mathrm{H}\right), 5.20\left(\mathrm{dq},-\mathrm{CH}_{2} \mathrm{CH}=\mathrm{CH}_{2}, J_{\text {cis }}=10.3 \mathrm{~Hz}, 1 \mathrm{H}\right), 5.25$ $\left(\mathrm{dq},-\mathrm{CH}_{2} \mathrm{CH}=\mathrm{CH}_{2}, J_{\text {trans }}=17.27 \mathrm{~Hz}, 1 \mathrm{H}\right), 5.85\left(\mathrm{~m},-\mathrm{CH}_{2} \mathrm{CH}=\mathrm{CH}_{2}, 1 \mathrm{H}\right), 6.475$ (br s, amide NHCO, 1H) ; HR-MS: m/z 176.98 (observed); 176.99 (calculated for $[\mathrm{M}+\mathrm{H}]^{+}$). 


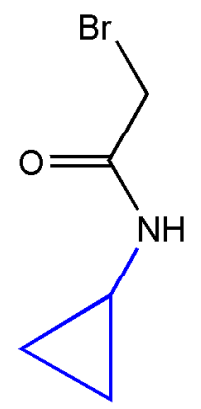

N-(cyclopropyl)-1-bromoethanamide: FT-IR: $3250 \mathrm{~cm}^{-1}$ (amide N-H str.), 2950-2850 cm-1 (C-H str.), $1680 \mathrm{~cm}^{-1}$ (Amide I, C=O str.), $1560 \mathrm{~cm}^{-1}$ (Amide II, N-H ben.), 1470-1410 cm $\mathrm{cm}^{-1}$ (C-C str.), 1290-1110 cm ${ }^{-1}$ (C-O str.); ${ }^{1} \mathrm{H}-\mathrm{NMR}\left(400 \mathrm{MHz}, \mathrm{CDCl}_{3}\right.$ ): $\delta / \mathrm{ppm} 0.565$ (m, cyCH $\left.\mathrm{CH}_{2}, 2 \mathrm{H}\right), 0.822$ (m, cyCH, $2 \mathrm{H}), 2.734(\mathrm{~m}, \mathrm{cyCH}, 1 \mathrm{H}), 3.8\left(\mathrm{~s},-\mathrm{COCH}_{2} \mathrm{Br}, 2 \mathrm{H}\right), 6.475$ (br s, amide $\left.-\mathrm{NHCO}, 1 \mathrm{H}\right)$ ); HR-MS: $m / z 177.98$ (observed): 177.98 (calculated for $[\mathrm{M}+\mathrm{H}]^{+}$).

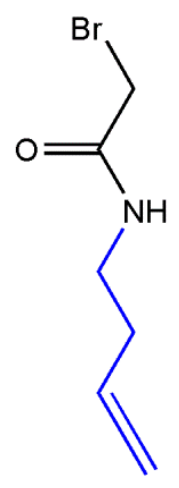

N-(but-3-enyl)-1-bromoethanamide: FT-IR: $3250 \mathrm{~cm}^{-1}$ (amide $\mathrm{N}-\mathrm{H}$ str.), $3049 \mathrm{~cm}^{-1}$ (C-H str.), $1680 \mathrm{~cm}^{-1}$ (Amide I, $\mathrm{C}=\mathrm{O}$ str.), $1642 \mathrm{~cm}^{-1}$ ( $\mathrm{C}=\mathrm{C}$ str.), $1560 \mathrm{~cm}^{-1}$ (Amide II, N-H ben.), 1470$1410 \mathrm{~cm}^{-1}$ (C-C str.), 1290-1110 cm ${ }^{-1}$ (C-O str.); ${ }^{1} \mathrm{H}-\mathrm{NMR}$ (400 MHz, $\mathrm{CDCl}_{3}$ ): $\delta / \mathrm{ppm} 2.3$ (m, $\left.\mathrm{CONHCH}_{2} \mathrm{CH}_{2^{-}}, 2 \mathrm{H}\right), 3.2\left(\mathrm{t},-\mathrm{CONHCH}_{2} \mathrm{CH}_{2^{-}}, 2 \mathrm{H}\right), 3.8\left(\mathrm{~s},-\mathrm{COCH}_{2} \mathrm{Br}, 2 \mathrm{H}\right), 5.138(\mathrm{dq}$, $\left.\mathrm{CH}_{2} \mathrm{CH}_{2} \mathrm{CH}=\mathrm{CH}_{2}, J_{\text {cis }}=10.52 \mathrm{~Hz}, 1 \mathrm{H}\right), 5.192\left(\mathrm{dq}, \mathrm{CH}_{2} \mathrm{CH}_{2} \mathrm{CH}=\mathrm{CH}_{2}, J_{\text {trans }}=17.27 \mathrm{~Hz}, 1 \mathrm{H}\right), 5.85$ (m, $\mathrm{CH}_{2} \mathrm{CH}_{2} \mathrm{CH}=\mathrm{CH}_{2}, 1 \mathrm{H}$ ), 6.475 (br s, amide $-\mathrm{NHCO}, 1 \mathrm{H}$ ) ; HR-MS: $m / z 190.98$ (observed); 190.99 (calculated for $[\mathrm{M}+\mathrm{H}]^{+}$). 


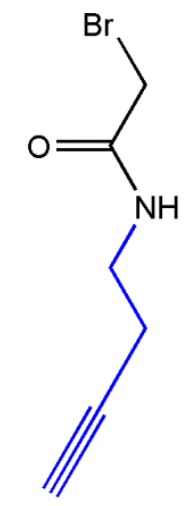

N-(but-3-ynyl)-1-bromoethanamide: FT-IR: $3250 \mathrm{~cm}^{-1}$ (amide N-H str.), $3310 \mathrm{~cm}^{-1}$ (三C-H str.), 2857-2942 $\mathrm{cm}^{-1}$ (-C-H str.), $2119 \mathrm{~cm}^{-1}$ (C $\equiv \mathrm{C}$ str.), $1680 \mathrm{~cm}^{-1}$ (Amide I, $\mathrm{C}=\mathrm{O}$ str.), $1642 \mathrm{~cm}^{-1}$ (C=C str.), $1560 \mathrm{~cm}^{-1}$ (Amide II, N-H ben.), $1470-1410 \mathrm{~cm}^{-1}$ (C-C str.), 1290-1110 cm ${ }^{-1}$ (C-O str.); ${ }^{1} \mathrm{H}-\mathrm{NMR}\left(400 \mathrm{MHz}, \mathrm{CDCl}_{3}\right): \delta / \mathrm{ppm} 1.82\left(\mathrm{t},-\mathrm{CH}_{2} \mathrm{C} \equiv \mathrm{CH}, 1 \mathrm{H}\right), 2.29\left(\mathrm{~m},-\mathrm{CONHCH}_{2} \mathrm{CH}_{2^{-}}\right.$, 2H), 3.37 (t, - $\left.\mathrm{CONHCH} \mathrm{CH}_{2}-, 2 \mathrm{H}\right), 3.8\left(\mathrm{~s},-\mathrm{COCH}_{2} \mathrm{Br}, 2 \mathrm{H}\right), 6.475$ (br s, amide $\left.-\mathrm{NHCO}, 1 \mathrm{H}\right)$ ); HR-MS: $m / z 188.98$ (observed); 188.97 (calculated for $[\mathrm{M}+\mathrm{H}]^{+}$). 


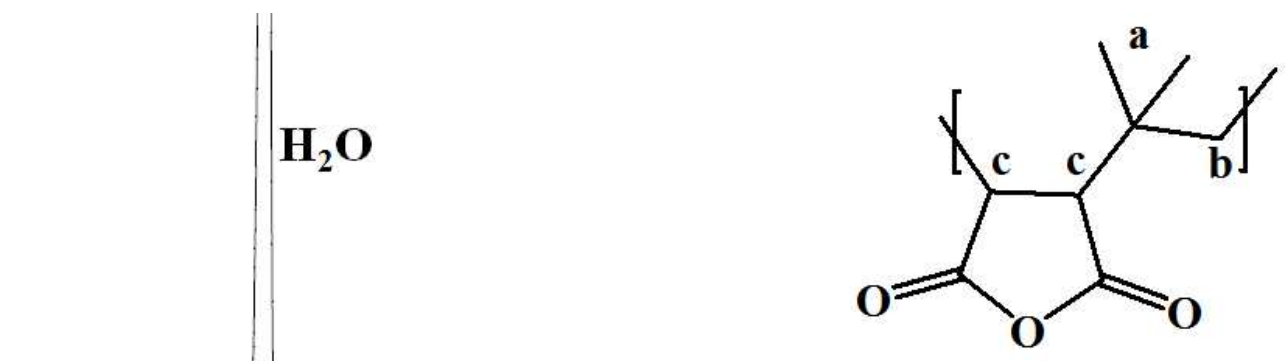

\section{$\left(\mathrm{CD}_{3}\right)_{2} \mathrm{SO}$}
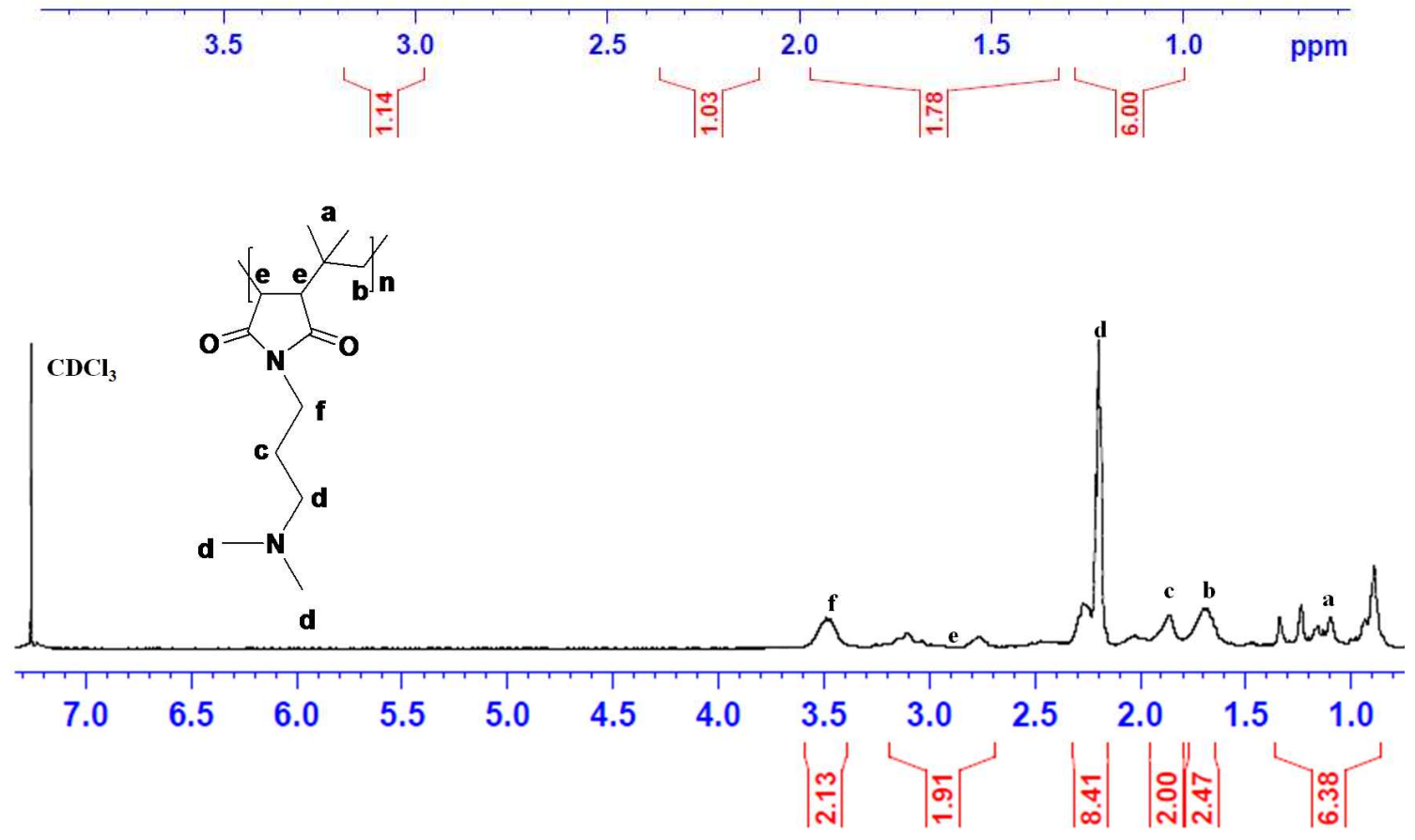

Figure S1. ${ }^{1} \mathrm{HNMR}$ spectra of precursor polymer, Poly(siobutylene-alt-maleic anhydride) in $\left(\mathrm{CD}_{3}\right)_{2} \mathrm{SO}$ and after conversion, PIBMI, poly(isobutylene-alt- $N-\left(N^{\prime}, N^{\prime}-\right.$ dimethylaminopropylmaleimide) in $\mathrm{CDCl}_{3}$. 


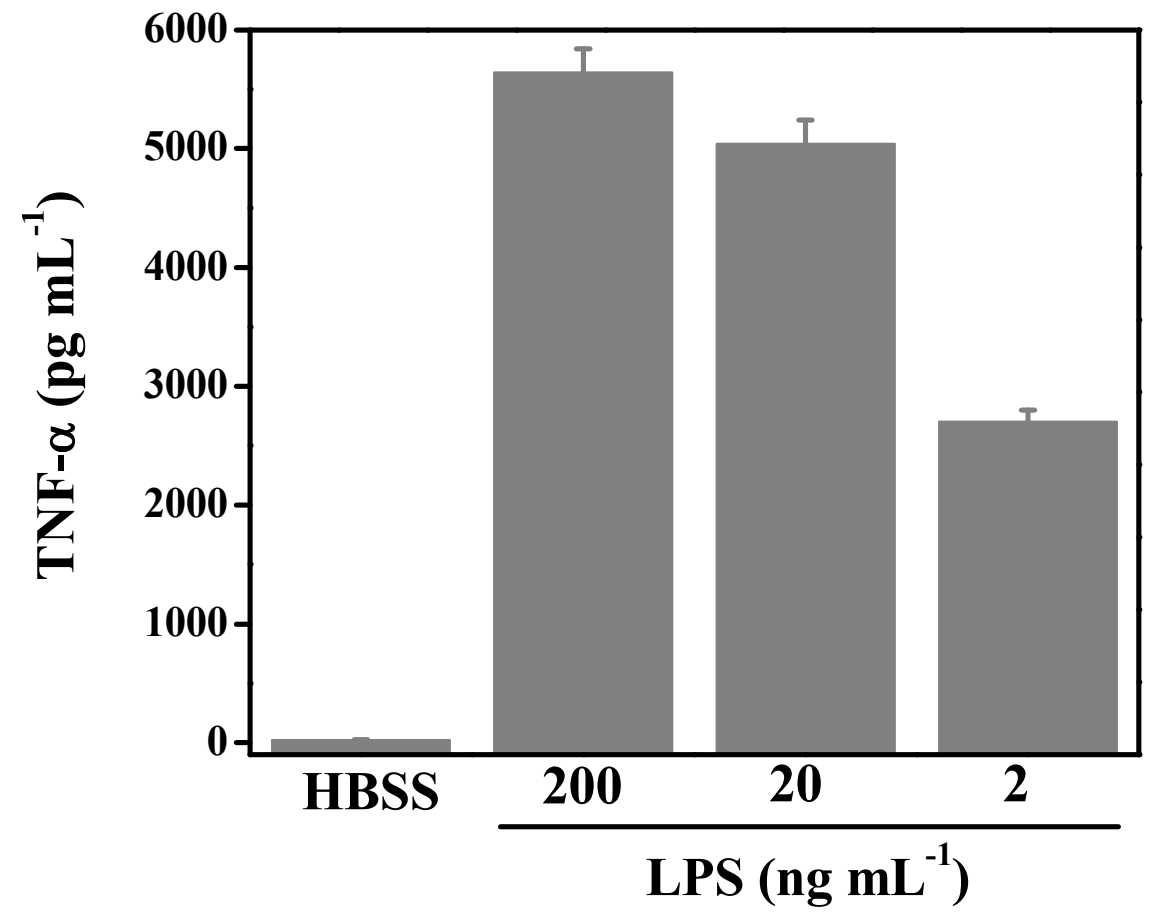

Figure S2. Stimulation of hPBMCs with LPS. Secretion of TNF- $\alpha$ after stimulation with different concentrations of LPS. 
(A)

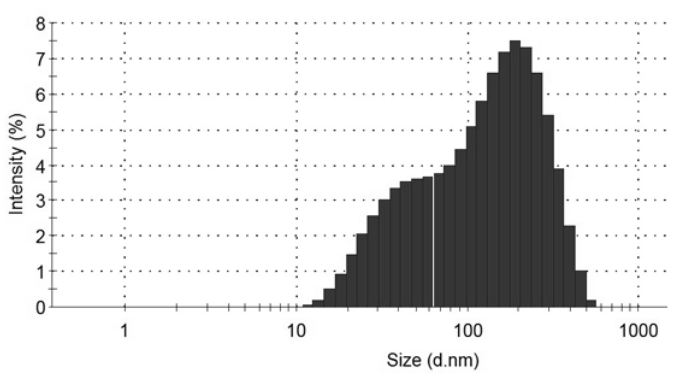

(B)

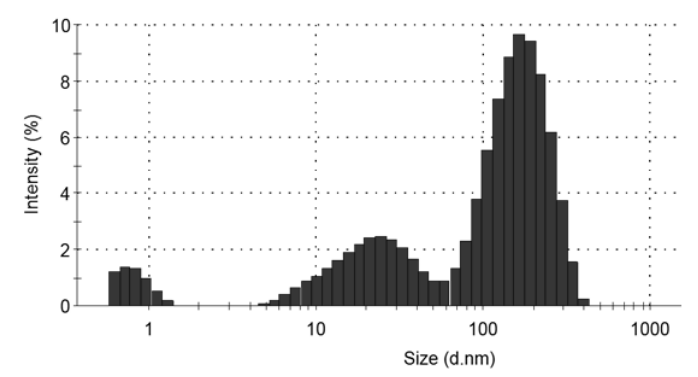

(C)

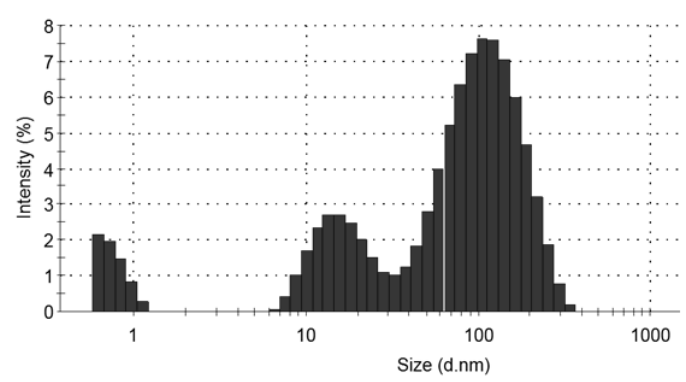

(D)

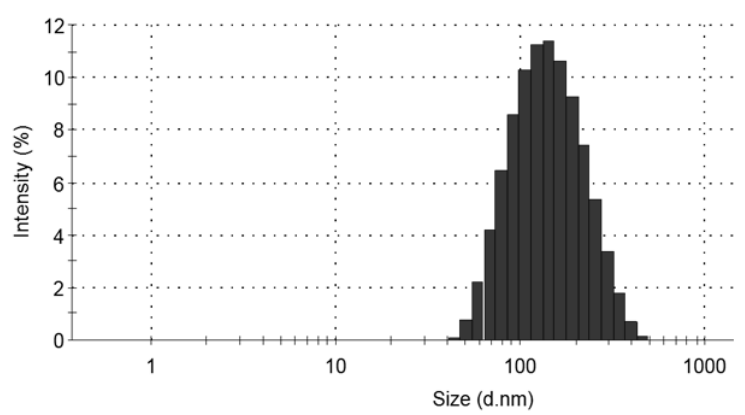

(E)

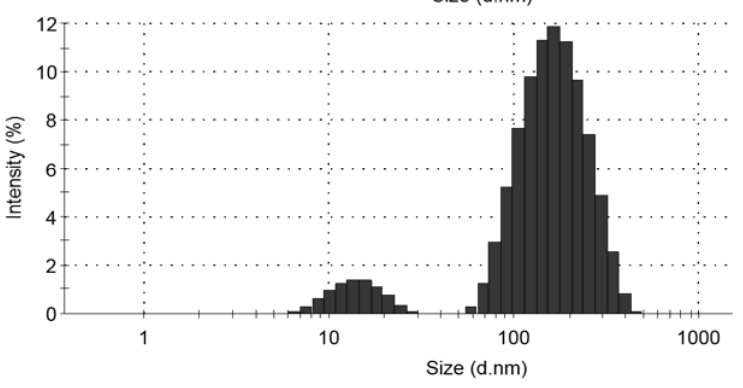

(F)

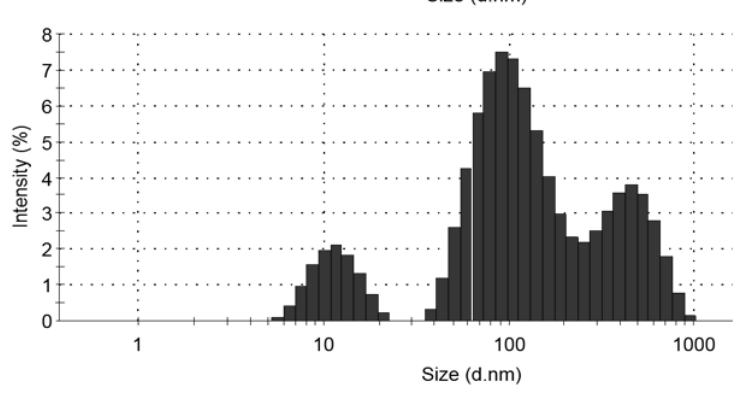

Figure S3. LPS binding studies of polymers using dynamic light scattering (DLS) studies. Size distribution of LPS (A), LPS + Q n-PenP (B), LPS + Qn-prEP, LPS + QCybuAP, LPS + Q nprAP and LPS + Q $n$-BEGP. LPS was used at a concentration of $12.5 \mu \mathrm{g} \mathrm{mL}^{-1}$ and LPS + polymer at $12.5 \mu \mathrm{g} \mathrm{mL}^{-1}+12.5 \mu \mathrm{g} \mathrm{mL}^{-1}$. 


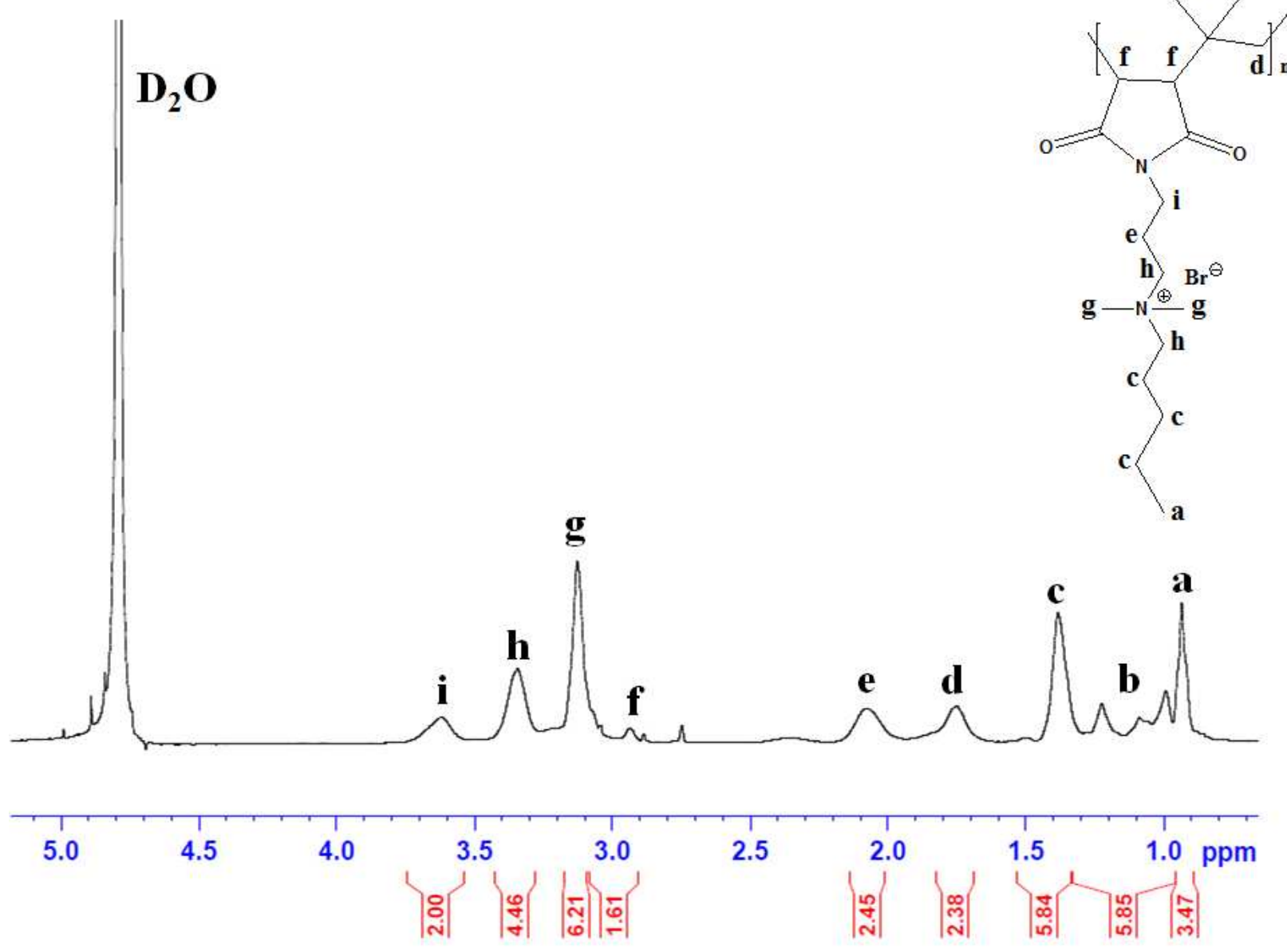




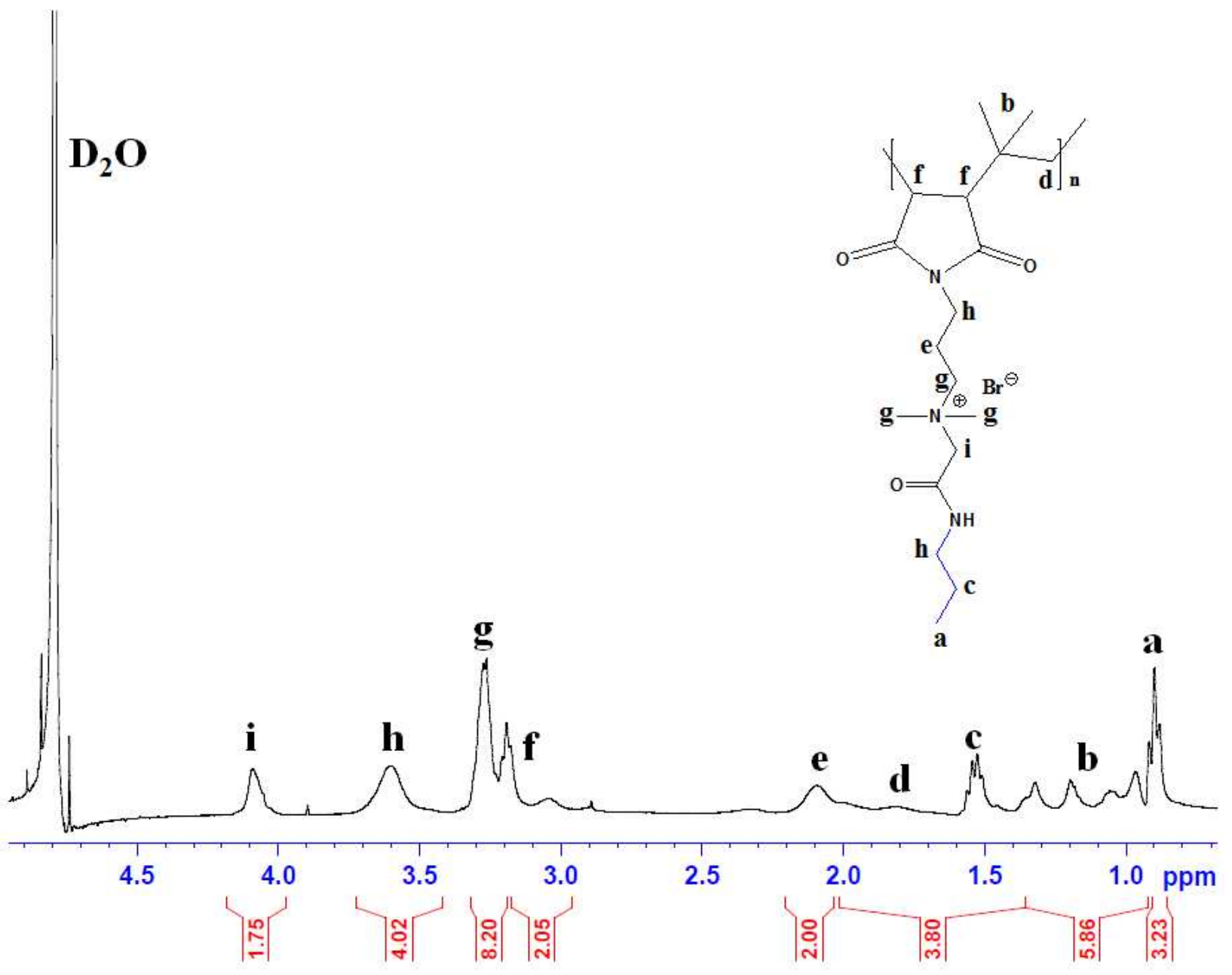




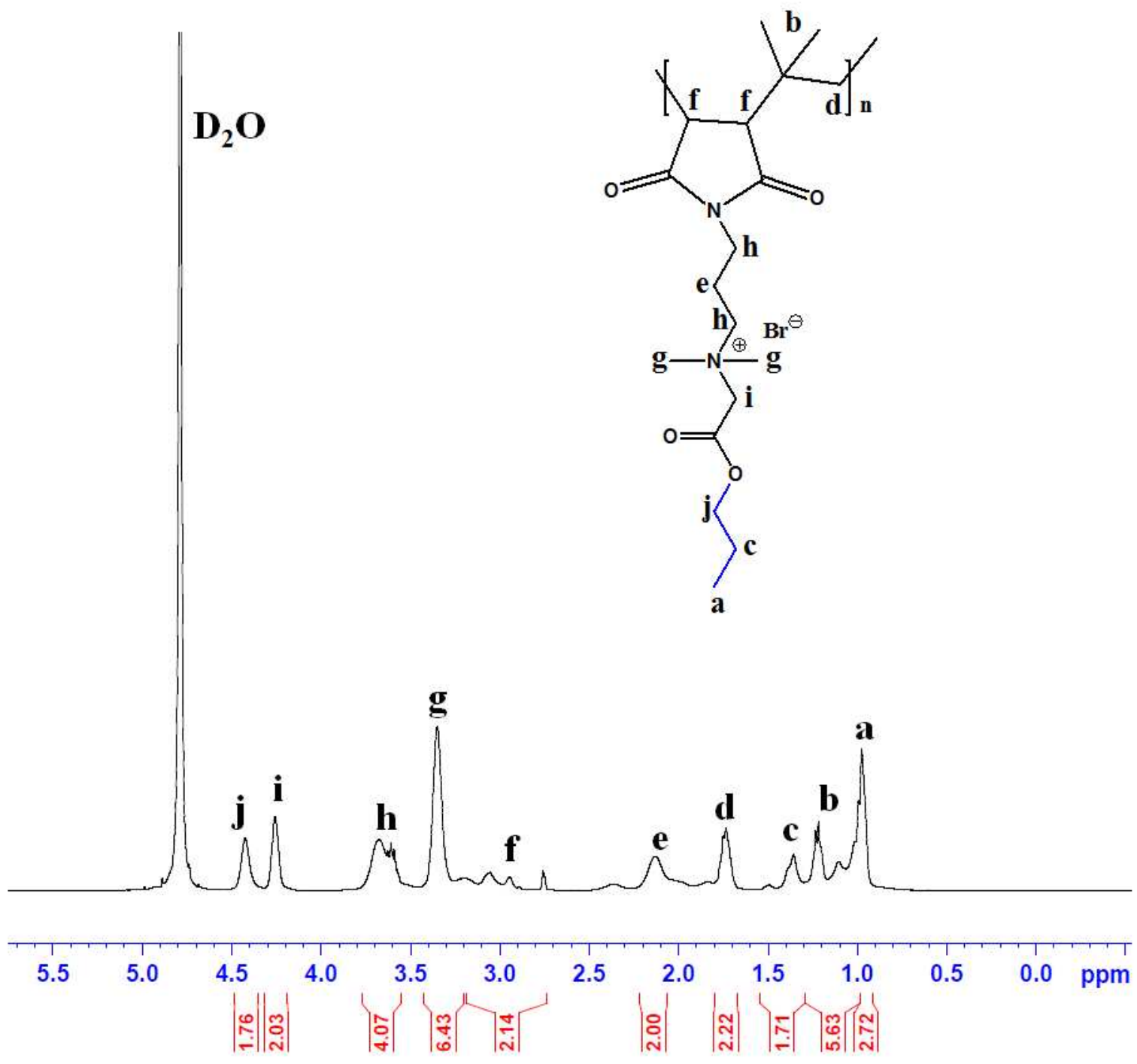




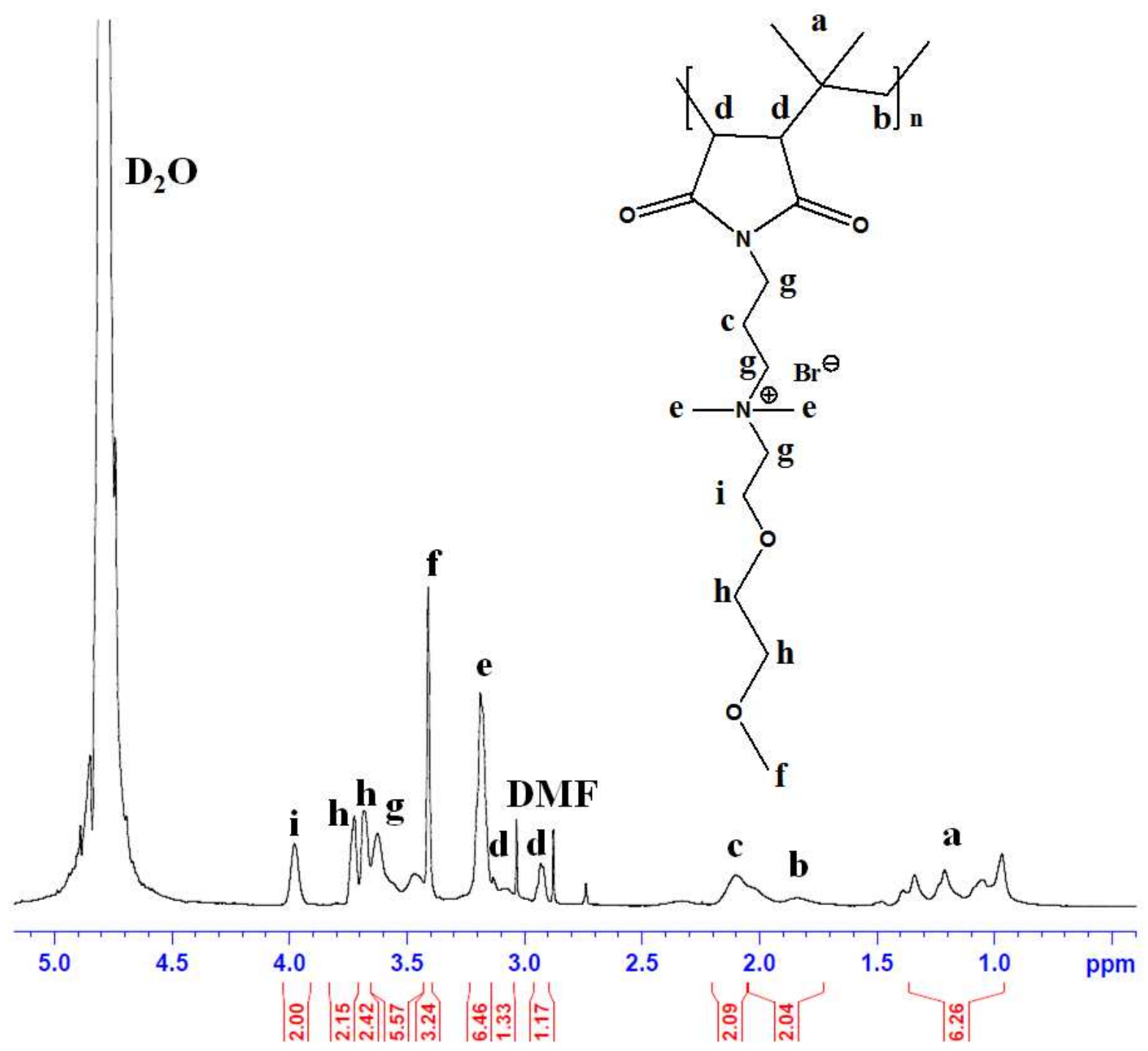




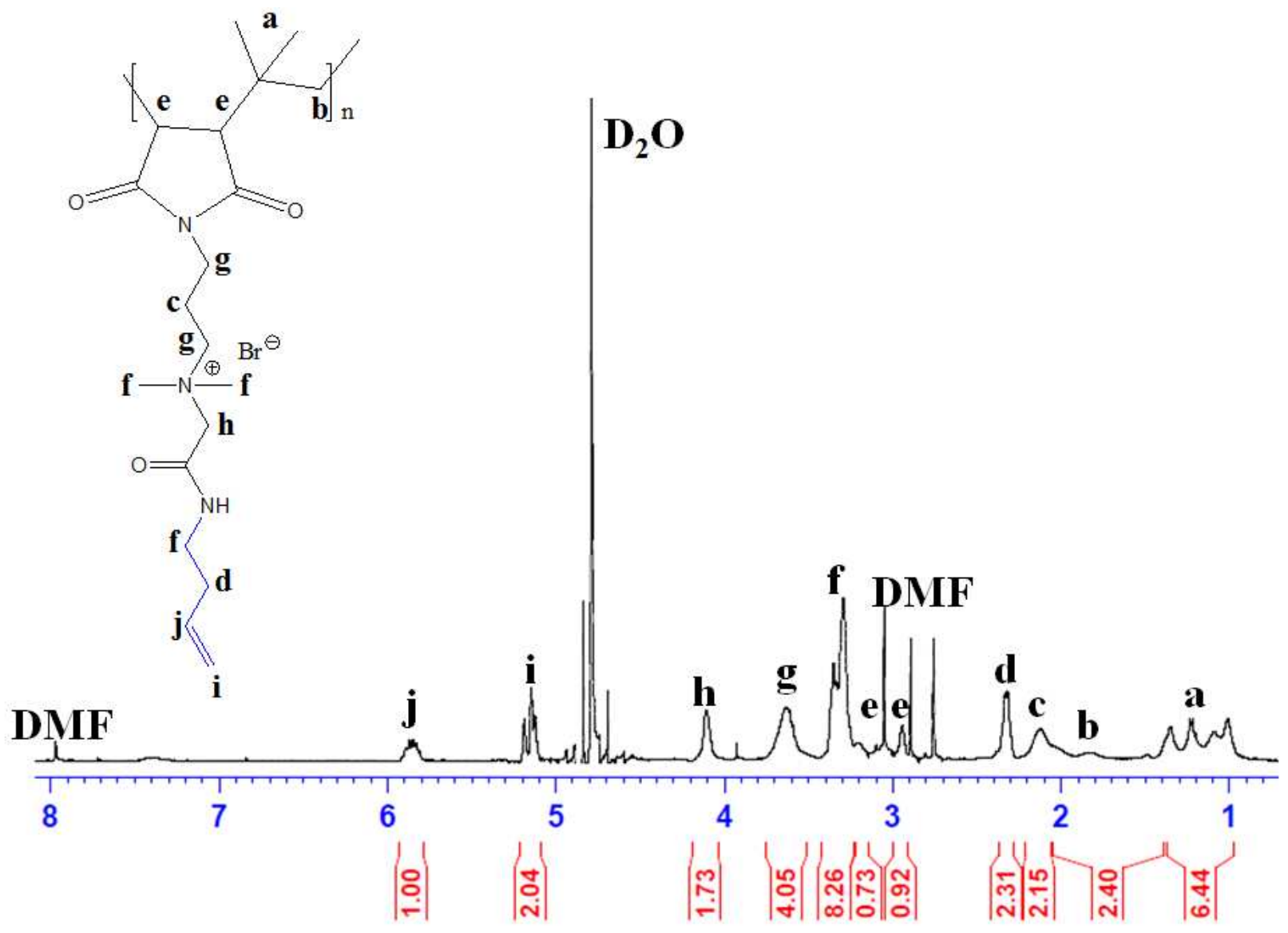




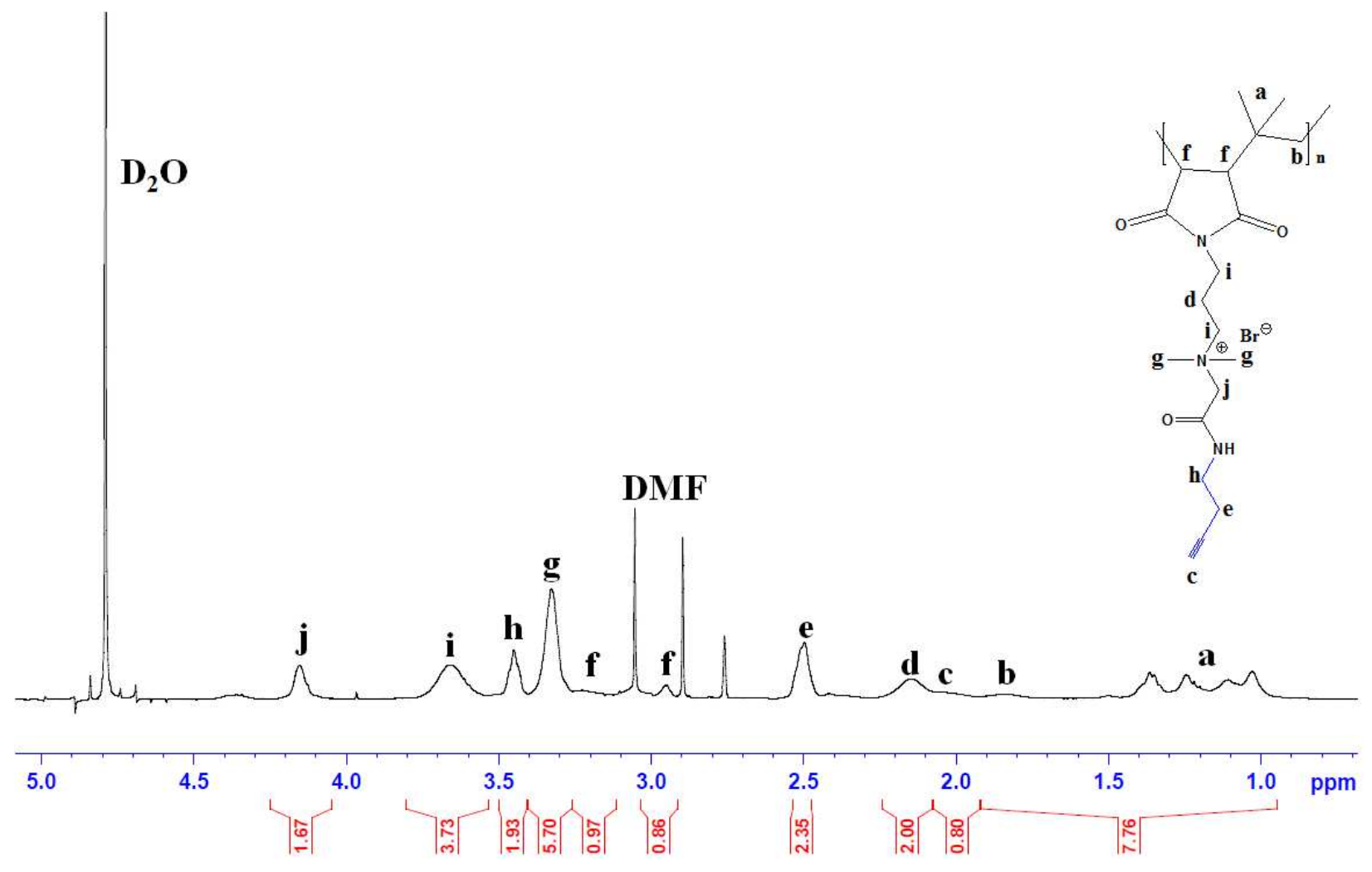




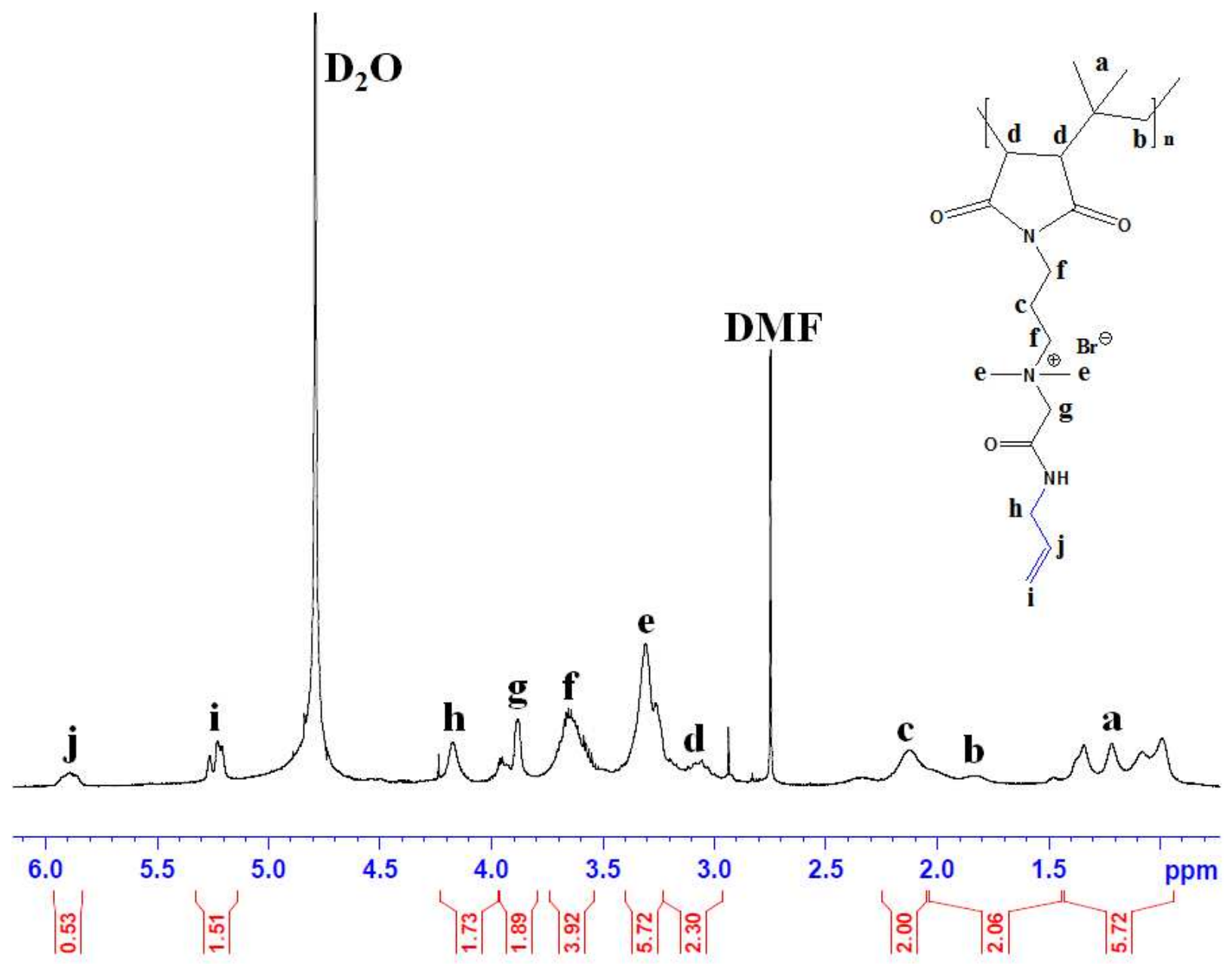




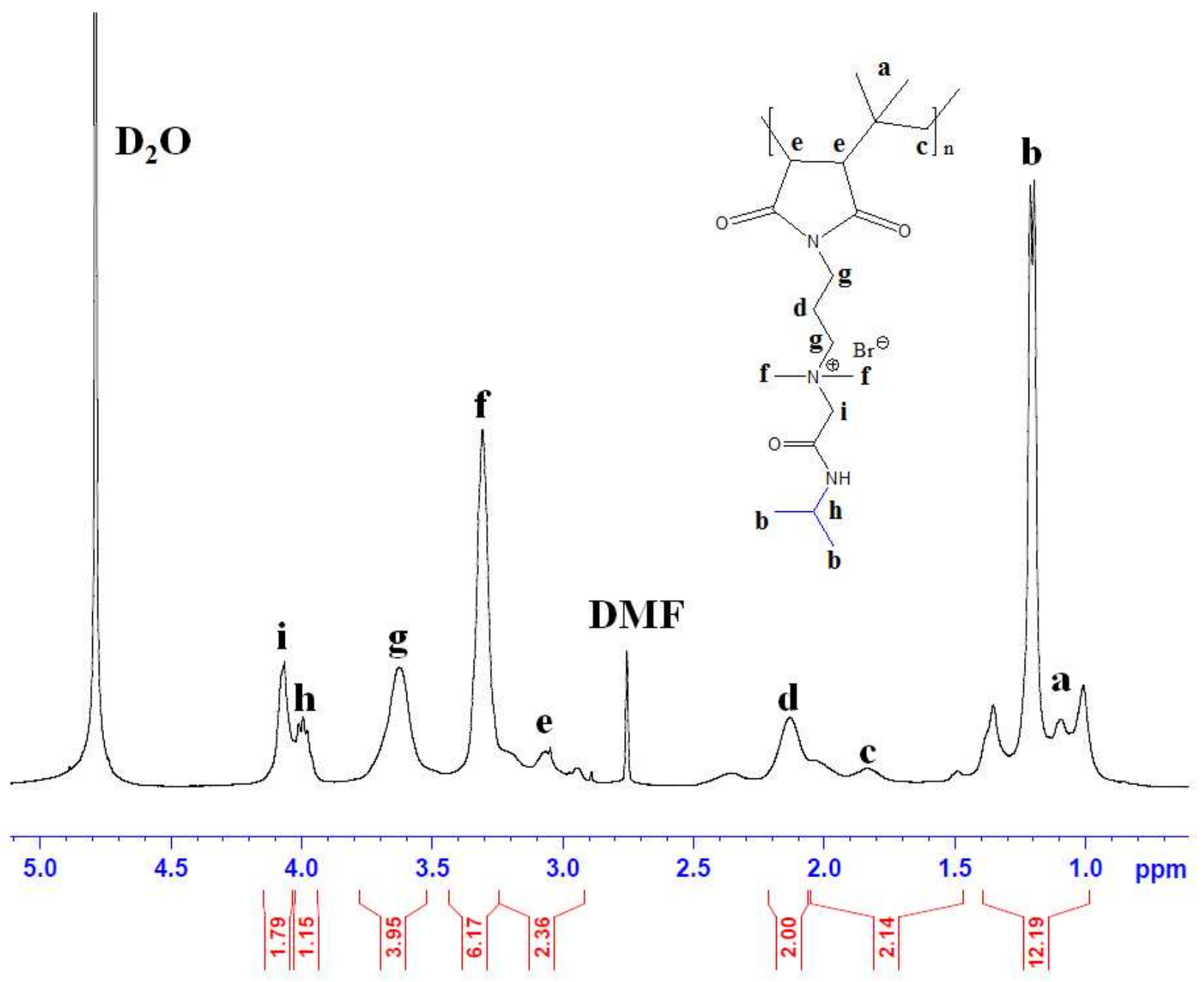




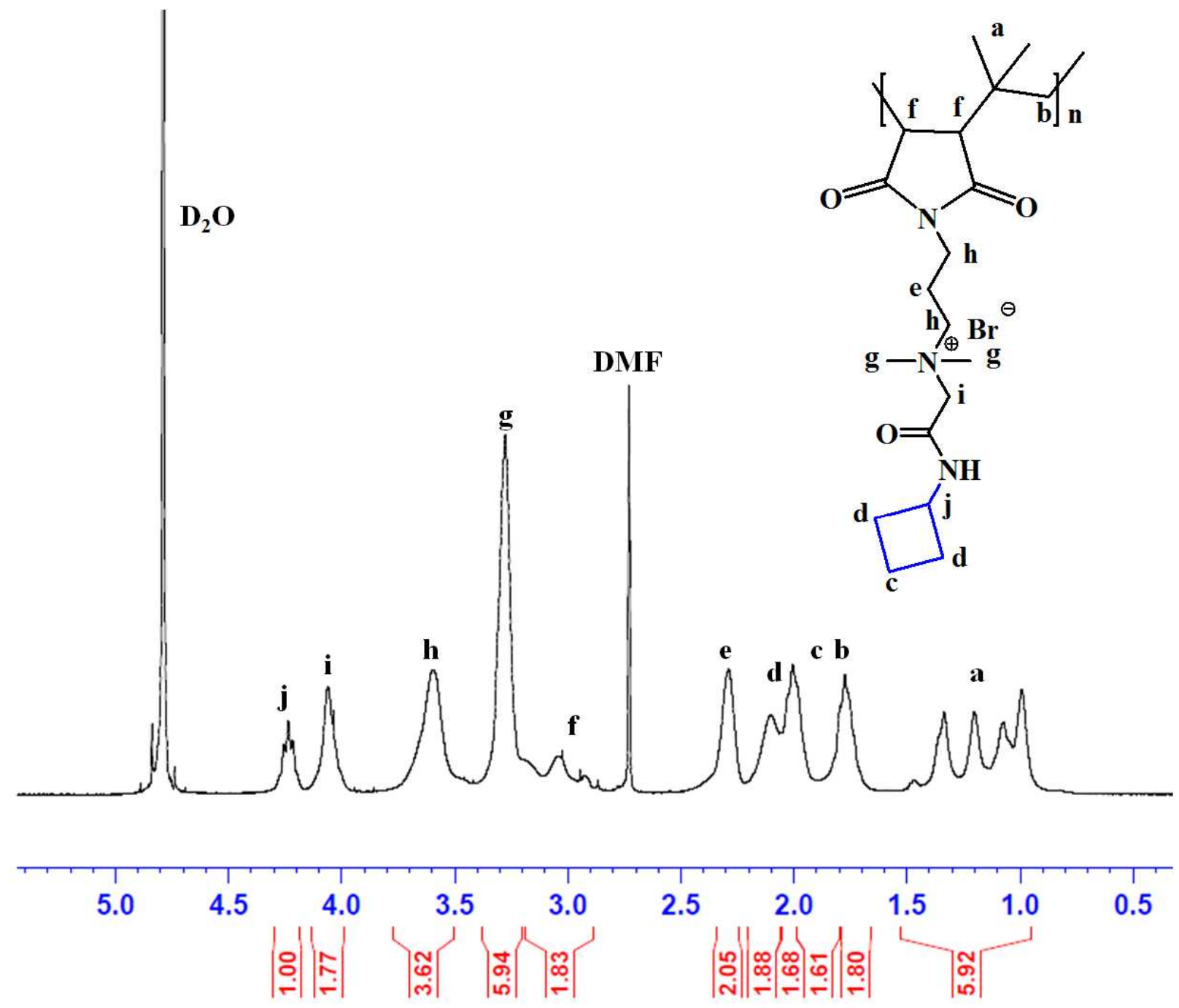




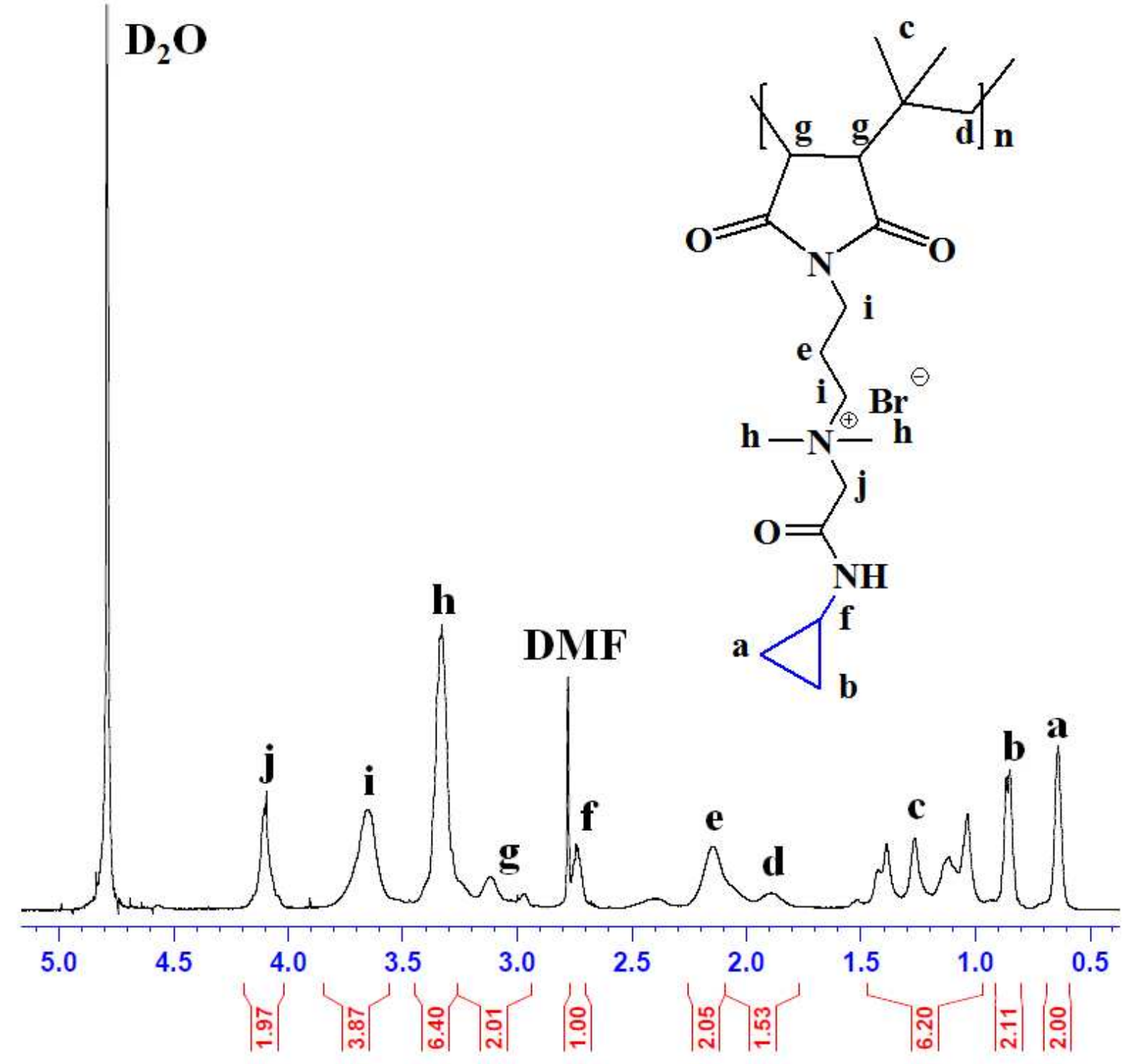

